\title{
Theoretical prediction of Jahn-Teller distortions and orbital ordering in $\mathrm{Cs}_{2} \mathrm{CuCl}_{2} \mathrm{Br}_{2}$
}

\author{
S.V. Streltsov ${ }^{1,2}$ and D.I. Khomskii ${ }^{3}$ \\ ${ }^{1}$ Institute of Metal Physics, S.Kovalevskoy St. 18, 620990 Ekaterinburg, Russia \\ ${ }^{2}$ Ural Federal University, Mira St. 19, 620002 Ekaterinburg, Russid ${ }^{\text {* }}$ \\ ${ }^{3}$ II. Physikalisches Institut, Universität zu Köln, Zülpicher Straße 77, D-50937 Köln, Germany
}

(Dated: October 13, 2018)

\begin{abstract}
With the use of the density function calculations we show that the actual crystal structure of $\mathrm{Cs}_{2} \mathrm{CuCl}_{2} \mathrm{Br}_{2}$ should contain elongated in the $a b$-plane $\mathrm{CuCl}_{4} \mathrm{Br}_{2}$ octahedra, in contrast to the experimentally observed compression in $c$-direction. We also predict that the spins on $\mathrm{Cu}^{2+}$ ions should be ferromagnetically ordered in $a b$-plane, while the exchange interaction along $c$-direction is small and its sign is uncertain.
\end{abstract}

PACS numbers: $75.25 .-\mathrm{j}, 75.30 . \mathrm{Kz}, 71.27 .+\mathrm{a}$

\section{INTRODUCTION}

The system $\mathrm{Cs}_{2} \mathrm{Cui}(\mathrm{Cl}, \mathrm{Br})_{4}$ attracts a lot of attention mainly because of its unconventional magnetic properties. $\mathrm{Cs}_{2} \mathrm{CuBr}_{4}$ is the only triangular-lattice antiferromagnet, which exhibits unusual quantum magnetization plateau, [1] while $\mathrm{Cs}_{2} \mathrm{CuCl}_{4}$ shows field-induced BoseEinstein condensation of magnons. 2] Thus the investigation of intermediate compositions of $\mathrm{Cs}_{2} \mathrm{CuCl}_{4-x} \mathrm{Br}_{x}$ may not only shed some light on the magnetic properties of both compounds, but may also results in the discovery of new phenomena.

The mixed $\mathrm{Cs}_{2} \mathrm{CuCl}_{4-x} \mathrm{Br}_{x}$ crystal series was successfully grown at $50{ }^{\circ} \mathrm{C}$ with orthorombic crystal structure. However, at room temperature in the synthesis process the stabilization of a new tetragonal phase for $1<x<2$ was observed. [3]

The crystal structure of tetragonal specimens consists of the $\mathrm{CuCl}_{2}$ layers stacking in the $c$-direction and divided by the $\mathrm{Cs}$ and $\mathrm{Br}$ atoms, see Fig. 1. It is important to mention that $\mathrm{Cu}$ ions in one of the layers placed on a top/bottom of the void between $\mathrm{CuCl}_{4}$ plaquettes of another layer. According ro Ref. 3, the $\mathrm{Cl}$ and $\mathrm{Br}$ ions form octahedra surrounding $\mathrm{Cu}$ with two short, apical, $\mathrm{Cu}-\mathrm{Br}$ and four long, planar, $\mathrm{Cu}-\mathrm{Cl}$ bonds. Such kind of the compressed octahedra are quite untypical for the JahnTeller $\mathrm{Cu}^{2+}$ ions with $d^{9}$ electronic configuration for the dense crystal structures, and the authors of Ref. 3 mentioned that the real type of distortions may be hidden by the multidomain structures.

There are a lot of insulating materials, where ligand octahedra surrounding Jahn-Teller active metal ion turn out to be elongated: $\mathrm{KCrF}_{4}$ [5, 6], $\mathrm{Cs}_{2} \mathrm{AgF}_{4}$ 7, 8], $\mathrm{K}_{2} \mathrm{CuF}_{4}[9$, 10], and others, while there are only few systems with the opposite distortion. Generally speaking there can be different mechanisms, which stabilize elongated octahedra. 11] One of the reasons can be the gain in the magnetic energy due to more efficient hoppings

*Electronic address: streltsov@imp.uran.ru between half-filled $x^{2}-y^{2}$-like and oxygen $p$-orbitals in the case of the $d^{9}$ configuration. Another one is related with the features of the elastic interactions. It can be shown that the total energy of elongated octahedra is lower than compressed if in the expression for the elastic energy the terms of higher order than quadratic are taken into account. [12]

In the present paper, using ab-initio band structure calculations, we found that the crystal structure of $\mathrm{Cs}_{2} \mathrm{CuCl}_{2} \mathrm{Br}_{2}(x=2)$, which corresponds to the lowest total energy, indeed corresponds to the elongated $\mathrm{CuCl}_{4} \mathrm{Br}_{2}$ octahedra. This is in contrast to the observed experimental structure, 3] but support general tendency in the Jahn-Teller distortions to stabilize elongated, not compressed octahedra. The more detailed structural study should be carried out to confirm (or disprove) the
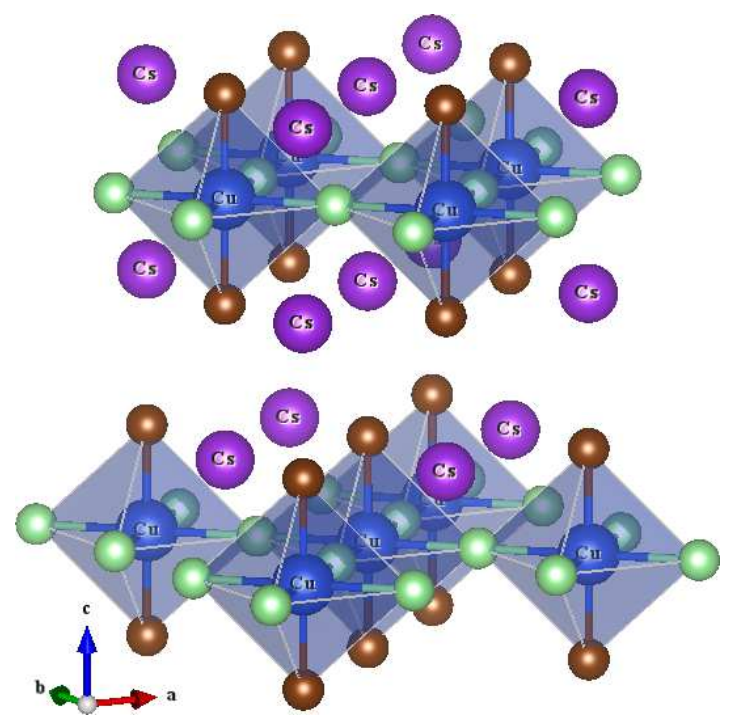

FIG. 1: (color online). The crystal structure of the tetragonal phase of $\mathrm{Cs}_{2} \mathrm{CuCl}_{2} \mathrm{Br}_{2}$. Blue balls are $\mathrm{Cu}$ ions, light green, brown and purple are $\mathrm{Cl}, \mathrm{Br}$ and $\mathrm{Cs}$ ions respectively. According to Ref. [3, the $\mathrm{CuCl}_{4} \mathrm{Br}_{2}$ octahedra are compressed along the c-axis. The image was generated using VESTA software. [4] 
TABLE I: Total energies in meV per formula unit for different magnetic configurations for the experimental crystal structure. [3] The energy of the FM configuration was chosen as zero.

\begin{tabular}{l|c}
\hline \hline & Total energy, meV \\
\hline FM & 0 \\
AFM-A & 3.7 \\
AFM-C & 2.9 \\
NM & 8.8 \\
\hline \hline
\end{tabular}

predicted lattice and orbital ordering.

\section{CALCULATION DETAILS}

The pseudo-potential PWscf code was chosen for the calculations. 13] We used ultrasoft pseudo-potentials with the nonlinear core correction and the Perdew-BurkeErnzerhof (PBE) version of the exchange-correlation potential. [14] In order to take into account strong Coulomb repulsion on the $\mathrm{Cu}$ sites the $\mathrm{GGA}+\mathrm{U}$ approximation was utilized. 15] On-site Coulomb repulsion parameter U was chosen to be $7.0 \mathrm{eV}$, while intra-atomic Hund's rule exchange $J_{H}=0.9 \mathrm{eV}$. 16

The charge density and kinetic energy cut-offs equal 40 Ry and $200 \mathrm{Ry}$, respectively. $144 k$-points $(6 \times 6 \times 4)$ in a full part of the Brillouin zone for the unit cell, consisting of 4 formula units (f.u.), were used in the self-consistency course. The structural optimization was performed while each component of the force were more than $2 \mathrm{mRy} / \mathrm{a} . \mathrm{u}$. No symmetry operations were used in the course of selfconsistency.

The crystal structure was taken for $\mathrm{Cs}_{2} \mathrm{CuCl}_{2.2} \mathrm{Br}_{1.8}$. 3 We used the supercell $\sqrt{2} \times \sqrt{2} \times 2$ cell to allow the simplest types of the orbital ordering.

\section{CALCULATION RESULTS}

The total energy is known to depend on the type of magnetic ordering, and this magnetic ordering may have an influence on the lattice distortions through the stabilization of the particular orbital ordering. 17] That is why it is important to study possible lattice distortions together with the analysis of the magnetic interactions.

The total energies of the following magnetic configuration were calculated: nonmagnetic (NM), ferromagnetic (FM), AFM-A type (when all ions in $a b$-plane are ferromagnetically ordered, while the interaction between planes is antiferromagnetic), and AFM-C type (nearest $\mathrm{Cu}$ in the $a b$-plane are AFM ordered, the next nearest neighbors in $c$-direction are ferromagnetically coupled).

The lowest in energy in the tetragonal crystal structure of Ref. 3 turns out to be the FM configuration (see Tab. I). The analysis of the occupation matrix shows

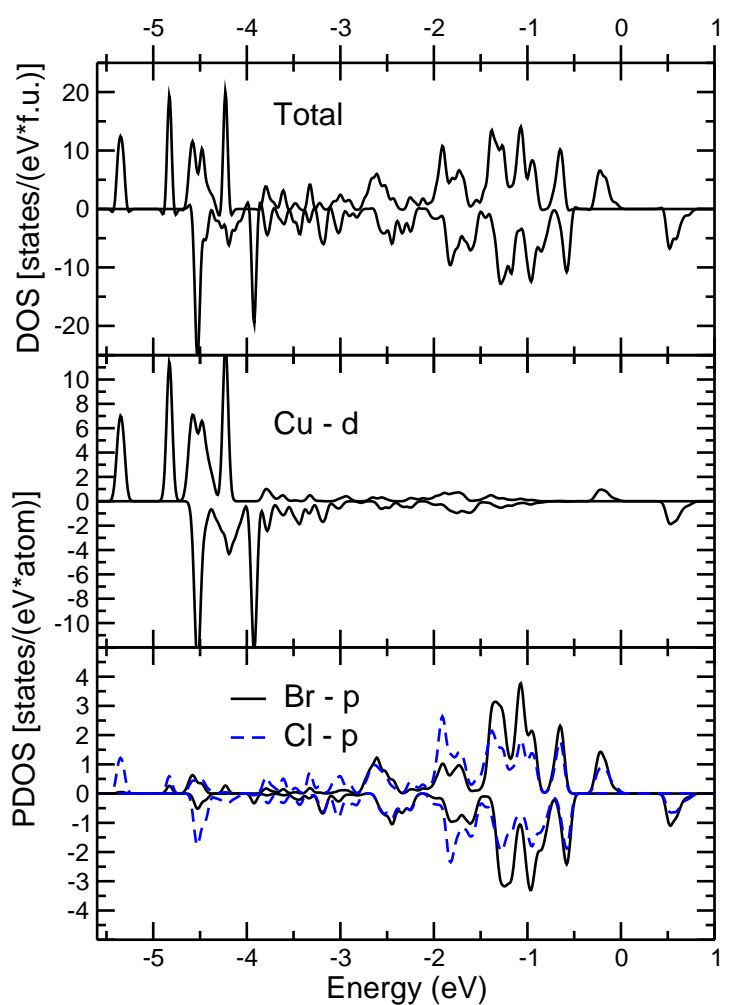

FIG. 2: (color online). The total and partial DOS for FM configuration in the relaxed crystal structure with alternating long and short $\mathrm{Cu}-\mathrm{Cl}$ bond in the $a b$-plane. Positive (negative) values correspond to spin majority (minority) states. The Fermi level is set to zero.

that the single hole in $3 d$-shell of $\mathrm{Cu}^{2+}$ is localized on $3 z^{2}-r^{2}$-orbital in the FM configuration. Such an orbital filling is obviously a result of the local compression of $\mathrm{CuCl}_{4} \mathrm{Br}_{2}$ octahedra in the structure of Ref. 3 .

The ground state is metallic for any of the investigated magnetic configurations. This is in contrast to the fact that the undoped materials $\mathrm{Cs}_{2} \mathrm{CuCl}_{4}$ and $\mathrm{Cs}_{2} \mathrm{CuBr}_{4}$ are Mott insulators [18, 19], and the samples of the intermediate compound $\mathrm{Cs}_{2} \mathrm{CuCl}_{2.4} \mathrm{Br}_{1.6}$ do not have metallic shine. 3]

The compression of the octahedra in $\mathrm{Cs}_{2} \mathrm{CuCl}_{2} \mathrm{Br}_{2}$ in the $c$-direction prevents the $x^{2}-z^{2} / y^{2}-z^{2}$ orbital order observed in $\mathrm{KCuF}_{3}$ [15] and $\mathrm{K}_{2} \mathrm{CuF}_{4}$ [9, [10], where $\mathrm{Cu}$ ions also have one hole in the $e_{g}$ sub-shell. In order to allow the same type of the orbital pattern and compare total energies of different solutions we tetragonally distorted $\mathrm{CuCl}_{4} \mathrm{Br}_{2}$ in the $a b$-plane and relaxed crystal structure with the constrain to keep the same cell volume. In addition we also performed the lattice optimization for the initial non-distorted in $a b$-plane structure, since the experimental structure not necessarily corresponds to the ground state crystal structure in the cho- 
TABLE II: Distances and total energies per formula unit for different magnetic configurations for the relaxed crystal structures. The energy of the FM configuration with two long and two short $\mathrm{Cu}-\mathrm{Cl}$ bonds was chosen as zero, it is $557.7 \mathrm{meV}$ lower than the FM solution for the tetragonal not relaxed crystal structure.

\begin{tabular}{l|c|c|c}
\hline \hline & Cu-Cl dist., $\AA$ & Cu-Br dist., $\AA$ & Total energy, meV \\
\hline FM & $3.02 \times 2 / 2.25 \times 2$ & $2.55 \times 2$ & 0 \\
FM & $2.64 \times 4$ & $2.45 \times 2$ & 285.1 \\
AFM-A & $3.02 \times 2 / 2.25 \times 2$ & $2.55 \times 2$ & -0.8 \\
AFM-C & $2.64 \times 4$ & $2.44 \times 2$ & 280.6 \\
NM & $2.64 \times 4$ & $2.44 \times 2$ & 286.4 \\
\hline \hline
\end{tabular}

sen approximation $(\mathrm{GGA}+\mathrm{U})$. In effect we obtained two crystal structures corresponding to the same magnetic order (relaxed initial experimental and relaxed distorted in ab-plane structures). The results are summarized in Tab. III, where the second, forth, and fifth lines refer to structure optimized staring from the lattice presented in Ref. 3 (four equal $\mathrm{Cu}-\mathrm{Cl}$ distances) and the first and the third lines correpond to the results for the structure with octahedra elongated in ab-plane.

The lowest total energy corresponds to FM and AFMA type configurations with strongly distorted in the $a b$-plane $\mathrm{CuCl}_{4}$ plaquettes, as shown in Fig. 3. The energy difference between these two solutions is tiny and may depend on the details of the calculations, but both lie much lower $(\sim 558 \mathrm{meV})$ in energy than the ground state magnetic configuration (FM) for the tetragonal not optimized crystal structure. Moreover, it is clearly seen from Tab. I that only half of this difference can be compensated by the ionic relaxation which does not change a local symmetry of $\mathrm{Cu}^{2+}$ ions (i.e. that there are 4 equal $\mathrm{Cu}-\mathrm{Cl}$ bonds in $a b$-plane).

The second half of the total energy decrease is related to the distortions in $a b$-plane such that they do not change the average $\mathrm{Cu}-\mathrm{Cl}$ bond length, but create checkerboard order of the long and short $\mathrm{Cu}-\mathrm{Cl}$ bonds. The distortions in the $a b$-plane are accompanied by a moderate elongation of the $\mathrm{CuCl}_{4} \mathrm{Br}_{2}$ octahedra in $c$-direction, $\mathrm{Cu}$-Br bond length increases on $\sim 0.1$ $\AA$, which is compensated by the Cs-Br bond squeezing. Thus, instead of compressed in $c$-direction octahedra, the ionic relaxation rather stabilizes the elongated in alternating directions in $a b$-plane $\mathrm{CuCl}_{4} \mathrm{Br}_{2}$ octahedra.

Such type of the distortions results in the $x^{2}-z^{2} / y^{2}-$ $z^{2}$ orbital order, like in $\mathrm{KCuF}_{3}$, see Fig. 3. This orbital pattern leads to a strong ferromagnetic super-exchange in $a b$-plane, which stabilizes FM or AFM-A magnetic configurations, which agrees with our total energy calculations.

Finally we present the total and partial density of states (DOS) plots for the distorted in $a b$-plane relaxed crystal structure, e.g. for FM configuration [Fig. 2)]. Due to a large on-site Coulomb repulsion $\mathrm{Cu}-3 d$ band goes

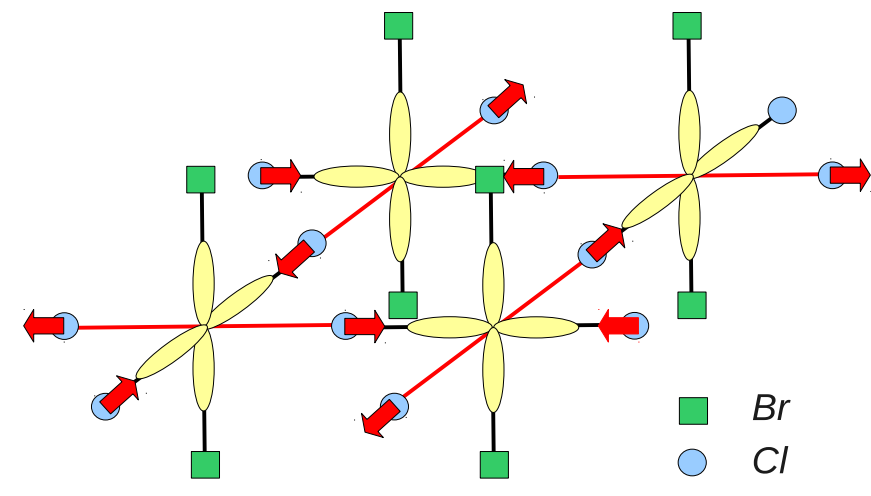

FIG. 3: (color online). The sketch of the proposed orthorombic distortions corresponding to the lowest total energy in Tab. III when all $\mathrm{CuCl}_{4} \mathrm{Br}_{2}$ octahedra are elongated in alternating directions in the $a b$ plane (long $\mathrm{Cu}-\mathrm{Cl}$ bonds are shown in red) and the orbital order, which is expected for such kind of the distortions (hole orbitals of $\mathrm{Cu}^{2+}$ are shown).

away from the Fermi level and is placed mainly in the energy range from $-5.5 \mathrm{eV}$ to $-3 \mathrm{eV}$. The $\mathrm{Br}-p$ and $\mathrm{Cl}-p$ states are concentrated from $-3 \mathrm{eV}$ to $1 \mathrm{eV}$. It is important to note, that in contrast to a naive expectation, the largest contribution to the bottom of the conduction band (as well as to the top of the valence band) comes from $\mathrm{Cl}-p$ and $\mathrm{Br}-p$ states: $\sim 3.6$ states $/\left[\mathrm{eV}^{*}\right.$ f.u. $]$, while $\mathrm{Cu}-d$ provides only 1.9 states $/\left[\mathrm{eV}^{*}\right.$ f.u. $]$ (the rest belongs to other states of $\mathrm{Cu}, \mathrm{Cl}$ and $\mathrm{Br}$ ions). This means that the hole is actually localized not on the $\mathrm{Cu}-x^{2}-y^{2}$ like orbital, but rather on the Wannier orbital, which is centered on the $\mathrm{Cu}$ site, has $x^{2}-y^{2}$ symmetry, but also has significant contributions (tails) on the surrounding $\mathrm{Cl}$ and $\mathrm{Br}$ ions. This is similar to the situation in $\mathrm{Cs}_{2} \mathrm{Au}_{2} \mathrm{Cl}_{6}, 20$ but here the spatial orientation of the Wannier orbital is different. The strong admixture of $\mathrm{Cl}-p$ and $\mathrm{Br}-p$ states to the conduction band may be the reason of the small band gap value $\sim 0.5 \mathrm{eV}$ in the present GGA $+U$ calculations, since these states act as a ballast. We expect that the $\mathrm{LDA}+\mathrm{U}_{W F}$ approximation, where $\mathrm{U}$ is applied not only on the $d$-part, but on a whole Wannier function will result in a larger band gap.

Summarizing, on the basis of the ab-initio calculations we have shown that the layered material $\mathrm{Cs}_{2} \mathrm{CuCl}_{2} \mathrm{Br}_{2}$, containing Jahn-Teller ion $\mathrm{Cu}^{2+}$, which was considered earlier as a rare example of the Jahn-Teller system with localized electrons and $e_{g}$ degeneracy with locally compressed ligand octahedra, must in fact have elongated octahedra with the long axes alternating in the basal plane. Thus, yet one more Jahn-Teller material turns out to be not an exception, but rather follows the general rule that the octahedra around such ions are elongated. This form of the Jahn-Teller distortions and orbital ordering should lead to strong ferromagnetic exchange in ab-plane, the interlayer exchange being very weak. The predicted crystal and magnetic structure should be observable by the de- 
tailed structural and magnetic studies.

\section{ACKNOWLEDGMENTS}

We thank R. Coldea who drew our attention to this system. This work is supported by the Russian Founda- tion for Basic Research via RFFI-10-02-96011 and RFFI10-02-00140, by the Ural branch of Russian Academy of Science through the young-scientist program and by the German projects SFB 608, DFG GR 1484/2-1, FOR 1346 .
[1] N. Fortune, S. Hannahs, Y. Yoshida, T. Sherline, T. Ono, H. Tanaka, and Y. Takano, Phys. Rev. Lett. 102, 257201 (2009), ISSN 0031-9007, URL http://link.aps.org/doi/10.1103/PhysRevLett.102.25720112

[2] T. Radu, H. Wilhelm, V. Yushankhai, D. Kovrizhin, R. Coldea, Z. Tylczynski, T. Lühmann, and F. Steglich, Phys. Rev. Lett. 95, 127202 (2005), ISSN 0031-9007, URL http://link.aps.org/doi/10.1103/PhysRevLett.95.127202

[3] N. Kruger, S. Belz, F. Schossau, A. A. Haghighirad, P. T. Cong, B. Wolf, S. Gottlieb-Schoenmeyer, F. Ritter, and W. Assmus, Crystal Growth \& Design 10, 4456 (2010), ISSN 1528-7483, URL http://pubs.acs.org/doi/abs/10.1021/cg100666t

[4] K. Momma and F. Izumi, J. Appl. Crystallogr. 44, 1272 (2011).

[5] G. Giovannetti, S. Margadonna, and J. van den Brink, Phys. Rev. B 77, 075113 (2008), ISSN 1098-0121, URL http://link.aps.org/doi/10.1103/PhysRevB.77.075113

[6] S. Margadonna and G. Karotsis, Journal of the American Chemical Society 128, 16436 (2006), ISSN 0002-7863, URL http://www.ncbi.nlm.nih.gov/pubmed/17177357

[7] H. Wu and D. Khomskii, Phys. Rev. B 76, 155115 (2007), ISSN 1098-0121, URL http://link.aps.org/doi/10.1103/PhysRevB.76.155115

[8] S. E. McLain, M. R. Dolgos, D. A. Tennant, J. F. C. Turner, T. Barnes, T. Proffen, B. C. Sales, and R. I. Bewley, Nature Materials 5, 561 (2006), ISSN 1476-1122, URL http://www.nature.com/doifinder/10.1038/nmat1670.

[9] K. Kugel and D. Khomskii, Zh. Eksp. Teor. Fiz 64, 725 (1973).

[10] Y. Ito and J. Akimitsu, J. Phys. Soc. Jpn. 40, 1333 (1976)
[11] J. Kanamori, Journal of Applied Physics 31, 14S (1960), URL http://ieeexplore.ieee.org/xpls/abs_all.jsp?arnumber $=51$ D. Khomskii and J. van den Brink, Phys. Rev. Lett. 85, 3329 (2000), URL http://link.aps.org/doi/10.1103/PhysRevLett.85.3329.

[13] P. Giannozzi, S. Baroni, N. Bonini, M. Calandra, R. Car, C. Cavazzoni, D. Ceresoli, G. L. Chiarotti, M. Cococcioni, I. Dabo, et al., J. Phys.: Condens. Matter 21, 395502 (2009).

[14] J. Perdew, K. Burke, and M. Ernzerhof, Phys. Rev. Lett. 77, 3865 (1996), ISSN 1079-7114, URL http://www.ncbi.nlm.nih.gov/pubmed/10062328.

[15] V. Anisimov, F. Aryasetiawan, and A. Lichtenstein, J. Phys.: Condens. Matter 9, 767 (1997), URL http://iopscience.iop.org/0953-8984/9/4/002.

[16] A. Liechtenstein and V. Anisimov, Physical Review B 52, 5467 (1995), URL http://prola.aps.org/abstract/PRB/v52/i8/pR5467_1

[17] K. I. Kugel and D. I. Khomskii, Soviet Physics Uspekhi 25, 231 (1982), ISSN 0042-1294.

[18] M.-A. Vachon, G. Koutroulakis, V. F. Mitrović, A. P. Reyes, P. Kuhns, R. Coldea, and Z. Tylczynski, J. Phys.: Condens. Matter 20, 295225 (2008), ISSN 0953-8984, URL http://stacks. iop.org/0953-8984/20/i=29/a=295225?key=cr

[19] K. Foyevtsova, Y. Zhang, H. O. Jeschke, and R. Valentí, Journal of Physics: Conference Series 145, 012038 (2009), ISSN 1742-6596, URL http://stacks.iop.org/1742-6596/145/i=1/a=012038?key=cr

[20] A. Ushakov, S. V. Streltsov, and D. I. Khomskii, J. Phys.: Condens. Matter 23, 445601 (2011), ISSN 1361-648X, URL http://www.ncbi.nlm.nih.gov/pubmed/22005022, 